\title{
Ameliorative potential of rutin on streptozotocin- induced neuropathic pain in rat
}

\author{
Maher M. Al-Enazi ${ }^{1,2}$ \\ ${ }^{1}$ Department of Medical Laboratory Sciences, College of Applied Medical Sciences, Salman bin Abdulaziz University, \\ Al-Kharj, Kingdom of Saudi Arabia. \\ ${ }^{2}$ Al Jouf University, Kingdom of Saudi Arabia.
}

Accepted 7 November, 2013

\begin{abstract}
The aim of the present study is to investigate the effect of rutin, a potent antioxidant and antiinflammatory compound on experimentally-induced diabetic neuropathy (DN) in male Wistar rats. In diabetic and normal rats, the pain-related behavior tests were performed before and after rutin (50 and $100 \mathrm{mg} / \mathrm{kg} / \mathrm{day}$ for 6 weeks) treatments. In serum, fasting glucose, insulin, tumor necrosis factor- $\alpha$ (TNF$\alpha$ ), interleukin-6 (IL-6) and interleukin-1 $\beta$ (IL-1 $\beta$ ) levels were estimated and in sciatic nerve, thiobarbituric acid reactive substances (TBARS), reduced glutathione (GSH) levels, and superoxide dismutase (SOD), catalase (CAT), glutathione-S-transferase (GST), glutathione-reductase (GR) and glutathione peroxidase (GSH-Px) activities were measured. Diabetic rats developed neuropathy which was apparent from decreased tail-flick and paw-withdrawal latencies and by following decrease in performance on Rota-Rod treadmill. Rutin treatments ameliorated the hyperalgesia, analgesia and improved motor coordination. Streptozotocin (STZ) significantly increased TBARS and decreased GSH levels in sciatic nerve where rutin treatment significantly protected those changes. In diabetic rats, SOD, CAT, GST, GSH-Px and GR activities were significantly inhibited. Rutin treatment significantly ameliorated decrease in antioxidant defense. Present results demonstrate protective effect of rutin in diabetic neuropathy through attenuation of oxidative stress and they suggest that rutin is a potential drug for the prevention of early diabetic-induced neuropathy.
\end{abstract}

Key words: Streptozotocin, rutin, oxidative stress, neuropathy, diabetes.

\section{INTRODUCTION}

World-wide, diabetic neuropathy (DN) is a major complication of diabetes mellitus. It affects about 15 to $25 \%$ in type- 1 and 30 to $40 \%$ in type- 2 diabetic patients, causing disabilities and a high mortality rate (Callaghan et al., 2012). Neuropathy pain is defined as a form of chronic pain that results from damage or abnormal function of central or peripheral nervous system (Abdi et al., 2004). The patients suffering from neuropathic pain frequently report sensory abnormalities such as burning sensations, hyperalgesia, allodynia and dysesthesia (Woolf, 2004). It can also alter the patient's quality of life by interfering with emotional well-being (Galer et al., 2000). Furthermore, it is a challenge in clinical practice, because of its severity, chronicity and resistance to some classical analgesics (Gilron et al., 2006).

Experimental studies in diabetic rats models have established that DN is the outcome of a complex network of unified vascular (Cameron and Cotter 1999), metabolic 
(Stevens et al., 2000) and neurotrophic (Calcutt et al., 2004) defects which conclude in electrophysiological discrepancies, abnormal sensory perception and progressive damage and loss of unmyelinated and myelinated nerve fibers (Sima et al., 2000). Diabetic neuropathies include several distinct syndromes of which symmetric sensory polyneuropathy, often associated with autonomic polyneuropathy is the most common and occurs in both type 1 and type 2 diabetes (Zochodne, 2008). Diabetic neuropathies accompanying type 1 diabetes tends to occur more predictably and to progress more rapidly, resulting in a more severe neuropathy (Sugimoto et al., 2000). Metabolic changes that may be involved in the pathogenesis of DN include polyol pathway flux, increased oxidative stress via glucose autoxidation and the subsequent formation of advanced glycation end products (AGEs), altered eicosanoid metabolism, activation of nuclear enzyme poly (ADPribose) polymerase and decreased antioxidant defense (Edwards et al., 2008). Oxidative stress is believed to be a biochemical trigger for sciatic nerve dysfunction and reduced endoneurial blood flow in diabetic rats (FigueroaRomero et al., 2008; Zherebitskaya et al., 2009). In this regard, the potential sources of reactive oxygen species (ROS) including endothelial nicotinamide adenine dinucleotide phosphate (NADPH) oxidase, xanthine oxidase, nitric oxide synthase and mitochondrial respiratory chain inefficiency are more notable (Cameron and Cotter 1999). Furthermore, diabetes is linked with reduced activity of reduced glutathione (GST), glutathione peroxidase (GSH-Px), glutathione-reductase (GR), Cu-Zn superoxide dismutase and lower levels of glutathione (Yu et al., 2006; Arora et al., 2008; Cui et al., 2008). Opposite to this, diabetes causes increase in the lipid peroxidation products such as malondialdehyde (MDA) or conjugated dienes in sciatic nerves (Cunha et al., 2008). Enhanced oxidative stress consecutively activates nuclear factor kappa B (NF-jB), which upregulates genes such as cytokines, adhesion molecules, endothelin-1 and tissue factor (Bierhaus et al., 1998).

Although DN is traditionally considered a nonimmune disease, accumulating evidence now indicates that immunologic and inflammatory mechanisms play a significant role in its development and progression (Tuttle, 2005; Mora and Navarro, 2006). TNF- $\alpha$ plays a major role in the immune system and it found increased in type 1 diabetic patients with DN and also showed independent correlation between interleukins and diabetic-induced neuropathy (Goldberg, 2009). Therefore, the present study is an attempt to estimate the levels of oxidative and inflammatory biomarkers in order to establish their association with experimentally-induced type 1 diabetes in rats.

Rutin (quercetin-3-rutinosid or vitamin-P) is a flavonol glycoside, which comprises of the flavonol quercetin and the disaccharide rutinose and it is known to have pharmacological activity such as lowering blood pressure and capillary reinforcement and also as an antiinflammatory (Ihme et al., 1996; Lindahl and Tangesson, 1997). Moreover, rutin has inhibitory effects against membrane lipid peroxidation (Lopez-Revuelta et al., 2006) and effectively suppressed adipocyte differentiation from pre-adipocytes (Choi et al., 2006). In addition, Park et al. (2002) showed that rutin can significantly decrease the thiobarbituric acid reactive substances (TBARS) content and increase the superoxide dismutase (SOD) activity in rat plasma suggesting possible protective role in oxidative stress-mediated diseases. Recently, Hao et al. (2012) documented the preventive effect of rutin on the development of $\mathrm{DN}$, which is closely related to oxidative stress and the TGF- $\beta_{1} / \mathrm{Smad} / \mathrm{ECM}$ and TGF$\beta_{1} / C T G F / E C M$ signaling pathways. These results suggest that rutin can prevent the development of experimental DN in rats.

Antioxidants such as quercetin, $n$-acetylcysteine and $\alpha$ lipoic acid have already progressed to clinical trials in DN, so we can speculate that rutin may pave its way for clinical trials in diabetic patients. Thus it is worth to experimentally examine the neuroprotective potential of rutin supplementation on the development of behavioral, biochemical and histopathological deficits in DN.

\section{MATERIALS AND METHODS}

\begin{abstract}
Animals
Male Wistar albino rats, roughly the same age of 3 months, weighing 180 to $200 \mathrm{~g}$ were received from the Experimental Animal Care Center (King Saud University, Riyadh, Saudi Arabia). They were maintained under controlled conditions of temperature $\left(22 \pm 1^{\circ} \mathrm{C}\right)$, humidity $(50$ to $55 \%)$, and light (12 h light/dark cycles) and were provided with Purina chow (Grain Silos \& Flour Mills Organization, Riyadh, Saudi Arabia) and water ad libitum (unless otherwise indicated during the experiment). All procedures including euthanasia procedure were conducted in accordance with the National Institute of Health Guide for the Care and Use of Laboratory Animals, Institute for Laboratory Animal Research (NIH Publications No. 80-23; 1996) and the Ethical Guidelines of the Experimental Animal Care Center (College of Pharmacy, King Saud University, Riyadh, Saudi Arabia).
\end{abstract}

\section{Diabetes induction}

Diabetes was induced in overnight fasted rats by a single intraperitoneal injection of streptozotocin (SIGMA Chemicals, USA) at a dose of $65 \mathrm{mg} / \mathrm{kg}$ body weight freshly dissolved in $0.1 \mathrm{~mol} / \mathrm{L}$ citrate buffer, pH 4.5 (Wang et al., 2013). Control rats as vehicle received equal volume of citrate buffer. Twenty five rats were injected with streptozotocin (STZ), from among them, 18 rats whose fasting blood glucose values were found more than $250 \mathrm{mg} / \mathrm{dl}$ after $72 \mathrm{~h}$ with no mortality were considered diabetic.

\section{Experimental design}

Normal healthy rats were divided in two groups (six rats in each 
group): (1) control (vehicle) and (2) rutin (100 mg/kg/day, gavage), and the STZ-induced diabetic rats were randomly divided as (3) diabetic (vehicle), (4) rutin (50 mg/kg/day, gavage) treated to diabetic rats and (5) rutin (100 mg/kg/day, gavage) treated to diabetic rats. Vehicle and drug treatment were started three weeks after the diabetes induction and continued for six consecutive weeks. Behavioral assessments were under taken before and after treatments.

\section{Mechanical hyperalgesia (Randall and Selitto method)}

Paw pressure thresholds were recorded with the paw pressure analgesia meter (MK-20D Analgesy meter, Muromachi KIKAI CO. Ltd., Japan). Pressure increasing at a linear rate of $10 \mathrm{~g} / \mathrm{s}$, with a cut-off at $250 \mathrm{~g}$ to avoid tissue injury, was applied to the center of the hindpaw. Similar technique has been used in our earlier study (Al-Enazi, 2013). When the animals displayed pain by withdrawal of the paw, the applied paw pressure was recorded by an analgesia meter and expressed as gram. Three tests separated by at least 10 min were performed for each rat, and mean of value is used.

\section{Tail flick test}

The method described by Sugimoto et al. (2008) and in our earlier study (Al-Enazi, 2013) was used with slight modifications. Acute nociception was induced by using a tail flick apparatus (Tail Flick model DS 20 Sorrel Apelex, France). Briefly, each rat placed in a restrainer and the tail flick latency was determined by focusing the intensity controlled beam of light on the distal last $2 \mathrm{~cm}$ of the animal's tail and recording the time taken to remove the tail from the noxious thermal stimulus. For each animal, 2 to 3 recordings were made at an interval of $15 \mathrm{~min}$; the mean value was used for statistical analysis.

\section{Rota-rod treadmill test}

Treadmill test was performed by using Rota-rod Treadmill for rats and mice (Model MK-670, Muromachi Kikai Co, Ltd., Tokyo, Japan) to evaluate motor coordination of the animals (Cartmell et al., 1991; Al-Enazi, 2013). Animals were initially trained to maintain themselves on the rotating rod for more than $2 \mathrm{~min}$. A day before treatment started and at the end of the treatment, the rats were placed on rotating rod for two trails each. Animals were scored for their latency to fall (in seconds) in each trial.

\section{Sample collections}

At the end of the treatment and behavioral assessments, animals were fasted overnight, under deep anesthesia, blood samples were collected through cardiac puncture and then they were sacrificed, and sciatic nerves were rapidly removed and dipped in liquid nitrogen for a minute and was kept in deep freezer at $-80^{\circ} \mathrm{C}$ till analysis. Blood samples were centrifuged at 3,000 rpm for $10 \mathrm{~min}$ and serum samples were stored at $-20^{\circ} \mathrm{C}$ till analysis.

\section{Serum parameters}

Serum fasting glucose levels were estimated by using commercially available kits (RANDOX Laboratories Ltd., UK) and insulin levels were measured by insulin enzyme immunoassay (ELISA) kit (DRG, Germany). Serum pro-inflammatory cytokines including tumor necrosis factor- $\alpha$ (TNF- $\alpha$ ), interleukin- 6 and $1 \beta$ (IL-6 and IL-1 $\beta$ ) concentrations were assayed by an enzyme-linked immunosorbent assay kit (ShangHai SenXiong Science and Technology Company, China). The levels were estimated by following the instruction provided by the manufacturer.

\section{Tissue parameters}

Sciatic nerves were homogenized in $50 \mathrm{mM}$ phosphate buffered saline ( $\mathrm{pH} 7.4$ ) by using a glass homogenizer (Omni International, Kennesaw, GA, USA). The homogenate was centrifuged at $1000 \mathrm{~g}$ for $10 \mathrm{~min}$ at $4^{\circ} \mathrm{C}$ to separate nuclei and unbroken cells. The pellet was discarded and a portion of supernatant was again centrifuged at $12000 \mathrm{~g}$ for $20 \mathrm{~min}$ to obtain post-mitochondrial supernatant. In homogenate, MDA and GSH levels were estimated. In postmitochondrial supernatant, SOD, catalase (CAT), GST, GSH-Px and GR activities were measured.

\section{Estimation of TBARS levels}

A TBARS assay kit (ZeptoMetrix) was used to measure the lipid peroxidation products, MDA equivalents. One hundred microliters of homogenate was mixed with $2.5 \mathrm{ml}$ reaction buffer (provided by the kit) and heated at $95^{\circ} \mathrm{C}$ for $60 \mathrm{~min}$. After the mixture had cooled, the absorbance of the supernatant was measured at $532 \mathrm{~nm}$ using a spectrophotometer. The lipid peroxidation products are expressed in terms of nmoles $\mathrm{MDA} / \mathrm{mg}$ protein using molar extinction coefficient of MDA-thiobarbituric chromophore $\left(1.56 \times 10^{5} / \mathrm{M} / \mathrm{cm}\right)$.

\section{Estimations of GSH levels}

The concentration of GSH was measured using the method described by Sedlak and Lindsay (1968). Homogenate was mixed with $0.2 \mathrm{M}$ Tris buffer, $\mathrm{pH} 8.2$ and $0.1 \mathrm{ml}$ of $0.01 \mathrm{M}$ Ellman's reagent, [5,5'-dithiobis-(2-nitro-benzoic acid)] (DTNB). Each sample tube was centrifuged at $3000 \mathrm{~g}$ at room temperature for $15 \mathrm{~min}$. The absorbance of the clear supernatants was measured using spectrophotometer at $412 \mathrm{~nm}$ in $1 \mathrm{~cm}$ quarts cells.

\section{Estimations of SOD activity}

The activity of SOD in sciatic nerve was estimated using the method described by Kono (1978) with the aid of nitroblue tetrazolium as the indicator. Superoxide anions are generated by the oxidation of hydroxylamine hydrochloride. The reduction of nitroblue tetrazolium to blue formazon mediated by superoxide anions was measured $560 \mathrm{~nm}$ under aerobic conditions. Addition of superoxide dismutase inhibits the reduction of nitroblue tetrazolium and the extent of inhibition is taken as a measure of enzyme activity. The SOD activity was expressed as units/mg protein.

\section{Estimation of CAT activity}

The CAT activity was measured by the method of Aebi (1984) using hydrogen peroxide as substrate in post-mitochondrial supernatant. The hydrogen peroxide decomposition by catalase was monitored spectrophotometrically (LKB-Pharmacia, Mark II, Ireland) by following the decrease in absorbance at $240 \mathrm{~nm}$. The activity of enzyme was expressed as units of decomposed $/ \mathrm{min} / \mathrm{mg}$ proteins by using molar extinction coefficient of $\mathrm{H}_{2} \mathrm{O}_{2}(71 / \mathrm{M} / \mathrm{cm})$. 


\section{Estimations of GST activity}

The GST activity in sciatic nerve was measured by the method of Habig et al. (1974). The reaction mixture consisted of $0.067 \mathrm{mM}$ GSH, $0.067 \mathrm{~nm}$ 1-chloro-2,4-dinitrobenzene (CDNB), $0.1 \mathrm{M}$ phosphate buffer $(\mathrm{pH} 6.0)$ and $0.1 \mathrm{ml}$ of post-mitochondrial supernatant in a total volume of $3 \mathrm{ml}$. Absorbance was read at 340 $\mathrm{nm}$ for $10 \mathrm{~min}$ every $30 \mathrm{~s}$ by an optical plate reader and the enzyme activity was calculated as mmol CDNB conjugate formed $\mathrm{min}^{-1} \mathrm{mg}^{-1}$ protein using a molar extinction coefficient of $9.6 \times 10^{3} \mathrm{M}^{-1} \mathrm{~cm}^{-1}$.

\section{Estimations of GSH-Px activity}

GSH-Px activity was assessed by modified method of Flohe and Gunzler (1984). For the enzyme reaction, $0.2 \mathrm{ml}$ of the postmitochondrial supernatant was placed into a tube and mixed with $0.4 \mathrm{ml}$ reduced glutathione and the mixture was put into an ice bath for $30 \mathrm{~min}$. Then the mixture was centrifuged for $10 \mathrm{~min}$ at 3000 $\mathrm{rpm}, 0.48 \mathrm{ml}$ of the supernatant was placed into a cuvette, and 2.2 $\mathrm{ml}$ of $0.32 \mathrm{M} \mathrm{Na}_{2} \mathrm{HPO}_{4}$ and $0.32 \mathrm{ml}$ of $1.0 \mathrm{mmol} / \mathrm{L}$ DTNB were added for color development. The absorbance at wavelength 412 $\mathrm{nm}$ was measured on spectrophotometer (LKB-Pharmacia, Mark II, Ireland) after $5 \mathrm{~min}$. The enzyme activity was calculated as $\mathrm{nmol} / \mathrm{mg}$ protein.

\section{Estimations of GR activity}

GR activity was measured in the post mitochondrial supernatant by the method of Carlberg and Mannervik (1985). GSSG is reduced to GSH by NADPH in the presence of GR. Enzyme activity was measured by following the decrease in absorbance (oxidation of NADPH) for $3 \mathrm{~min}$ spectrophotometrically at $340 \mathrm{~nm}$. The activity of enzyme was expressed as nmoles NADPH oxidized $/ \mathrm{min} / \mathrm{mg}$ protein, using molar extinction coefficient of NADPH (6.22; 106/M/cm).

\section{Histopathological screening of sciatic nerve}

A part of sciatic nerve was fixed in $10 \%$ neutral buffered formalin, embedded in paraffin wax, sectioned at $3 \mu \mathrm{m}$, stained with Hematoxylin and Eosin (H\&E) stain and placed in slides for under light microscopic examination.

\section{Statistical analysis}

Data were expressed as means \pm standard error of mean (SEM). Statistical analysis was carried out using one-way analysis of variance (ANOVA) followed by Newman-Keuls post-hoc test. $P$ value of $\leq 0.05$ was considered statistically significant. All statistics tests were conducted using Graph Pad Prism (version 5) software.

\section{RESULTS}

Mean body weights are significantly decreased in rats 9 weeks after the STZ injection. Rutin treatment with higher dose significantly increased the body weights compared to untreated diabetic animals (Figure 1).

Plasma fasting glucose levels significantly increased while insulin levels were decreased in STZ-induced diabetic rats. Treatments with rutin (50 and $100 \mathrm{mg} / \mathrm{kg} /$ day) to diabetic animals for 6 consecutive weeks showed significant decrease in fasting glucose and increase in insulin levels when compared with untreated diabetic rats (Figure 2).

In paw pressure analgesia test, vehicle-treated diabetic rats exhibited significantly decreased paw withdrawal latency (PWL) compared to non diabetic animals. The diabetic group of animals treated with rutin at both doses (50 and $100 \mathrm{mg} / \mathrm{kg} /$ day) for 6 weeks significantly increased the PWL time(s) compared to untreated diabetic rats (Figure $3 \mathrm{~A}$ ).

A significant decrease in tail flick latency was observed after 9 weeks of STZ-induced diabetic rats compared to control group. This decrease in tail flick response latency was significantly reversed on treatments with rutin low and high dose, respectively (Figure 3B).

Rota-rod treadmill performance of diabetic and nondiabetic animals, before and after treatment with two doses of rutin is as shown in Figure 3C. The running performance on treadmill was significantly decreased in diabetic animals as compared to the control rats. Rutin treatments to diabetic rats significantly enhanced the performance in dose dependent manner.

Serum pro-inflammatory markers including TNF- $\alpha$, IL$1 \beta$ and IL6 levels were markedly increased in diabetics as compared to control rats. Rutin treatments at both doses during six consecutive weeks in diabetic rats significantly decreased the levels of TNF- $\alpha$ and interleukins (Figure 4A to $C$ respectively).

Lipid peroxidation bio-marker, TBARS levels were significantly increased in the sciatic nerves of diabetic rat as compared to control group (Figure 5A), while GSH levels were significantly decreased in same group of animals (Figure 5B). Treatments with rutin, significantly decreased the TBARS levels and significantly prevented the decrease in GSH levels in sciatic nerves of diabetic rats (Figure 5A and $B$ ).

In sciatic nerves, SOD, CAT, GST, GSH-Px and GR activities were significantly decreased in diabetic animals as compared to the control ones (Figure 6). In diabetic rats, these activities were significantly enhanced by the daily treatment with rutin during six consecutive weeks. In control group, microscopic feature of transverse section of sciatic nerve showed benign looking with even distributed axons with its myelin sheath (Figure 7A). In transverse section of sciatic nerve of diabetic rat, partial and focal peripheral axonal loss, early regenerative process in the form of regenerating clusters of thinly myelinated axons with originally Schwann cell's basal lamina and few scattered degenerated bodies were found. Partial axonal loss also was noticed with longitudinal section of nerve (Figure 7B). In low doses rutin-treated rats, transverse section of sciatic nerve showed focal scattered peripheral axons loss, very few perineural scattered inflammatory cells infiltrate composed of small lymphocytes and also few scattered 


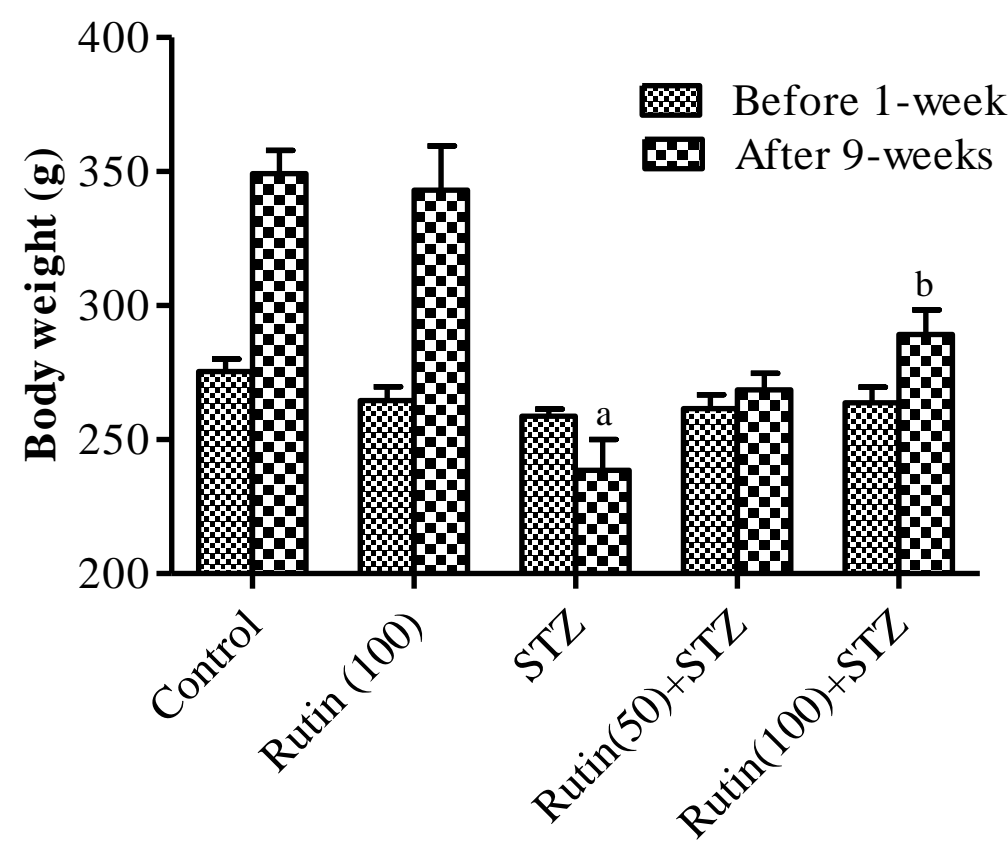

Figure 1. Effect of rutin on body weights of diabetic and non-diabetic animals at the start and at the end of the study. One-way ANOVA and Student-Newman-Keuls multiple comparisons test was applied. aSignificantly different from control group $(P<0.05)$ and ${ }^{b}$ Significantly different from STZ group $(P<0.05)$. Values are expressed as Mean $\pm S E M$ and six rats were used in each group.

\section{Glucose}

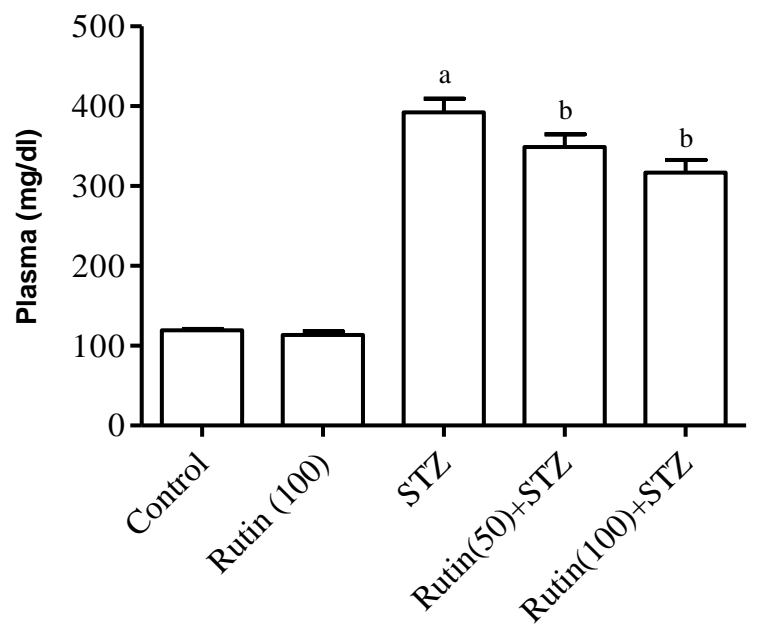

Insulin

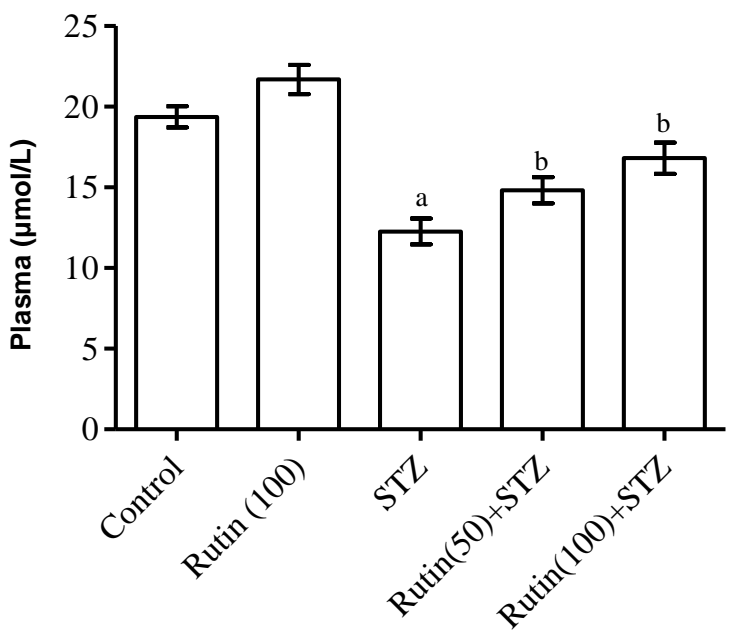

Figure 2. Effect of rutin on plasma glucose and insulin levels of diabetic and non-diabetic animals. One-way ANOVA and Student-Newman-Keuls multiple comparisons test was applied. 'a' Significantly different from control group $(P<0.05)$ and 'b' Significantly different from STZ group $(P<0.05)$. Values are expressed as Mean \pm SEM and six rats were used in each group.

lipoid degeneration of axons was seen (Figure 7C). However, in higher doses, rutin treatment showed benign normal looking with even distributed axons having its myelin sheath with congested blood vessels in transverse 

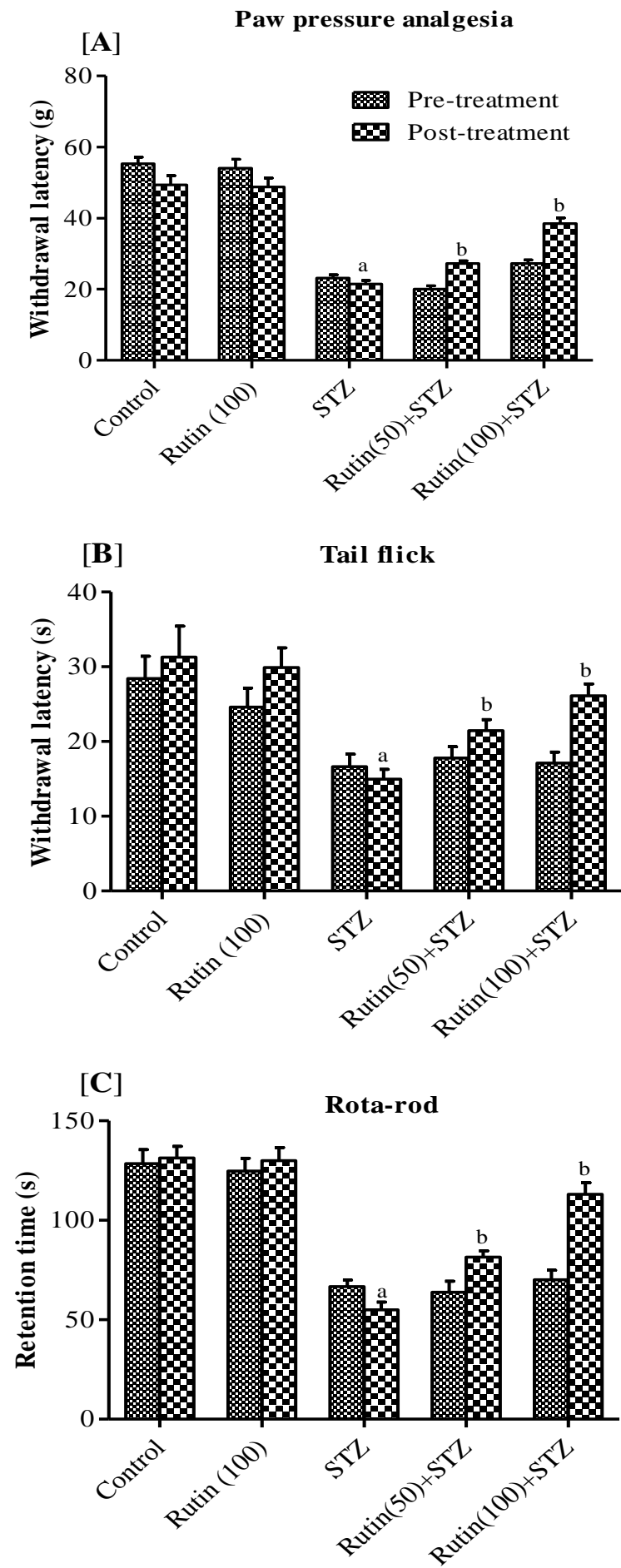

Figure 3. Effect of rutin on pain threshold in paw pressure analgesia, tail flick and Rota-rod treadmill performance of diabetic and non-diabetic animals. Oneway ANOVA and Student-Newman-Keuls multiple comparisons test was applied.

a Significantly different from control group $(P<0.05)$ and ${ }^{b}$ Significantly different from STZ group $(P<0.05)$. Values are expressed as Mean \pm SEM and six rats were used in each group. section of sciatic nerve (Figure 7D).

\section{DISCUSSION}

Neuropathic pain is one of the most common complications of diabetes mellitus. Along the disease course, almost $50 \%$ of the diabetic patients develop neuropathy with symptoms including spontaneous pain, allodynia and hyperalgesia (Apfel et al., 2001). STZinduced diabetic animals are used to model chronic neuropathic pain with hyperalgesia and allodynia that reflect symptoms observed in diabetics (Gul et al., 2000; Kamei et al., 2001). In the present study, in diabetic rats, the tail withdrawal latency was significantly shorter than that observed in control animals, indicating development of thermal hyperalgesia. This was accompanied by decreased motor coordination as assessed by performance on Rota-rod treadmill. The present results are in good agreement with the literature which frequently reports on hypersensitivity to mechanical stimulation (Chen and Levine, 2001; Dobretsov et al., 2003). A decrease of the pressure withdrawal threshold by 30 to $40 \%$ after 3 weeks of STZ-diabetes is reported by Romanovsky et al. (2004). Increased nociceptor activity and sensitivity during hyperglycemic hypoxia could be a mechanism e.g. burning pain attacks in painful neuropathy (Fuchs et al., 2010). Most of the phenolic compounds are known to have anti-inflammatory, analgesic and also have antinociceptive properties (Kamboj et al., 2010; Ramirez et al., 2010; Lee et al., 2006). This may be because of rutin treatment to diabetic rats for 6 consecutive weeks showed significant improvement in tail withdrawal latency rate as compared to untreated diabetic animals in the present study. Shen et al. (2002) and Alsaif (2009) have reported that, rutin has anti-inflammatory and analgesic properties.

Tumor necrosis factor is a cytokine that is involved in systemic inflammation and is a member of a group of cytokines that stimulate the acute phase reaction (Locksley et al., 2001). The promoter polymorphism in the TNF gene has been implicated in the regulation of TNF- $\alpha$ production and has been associated with a wide spectrum of inflammatory and infectious diseases. Furthermore, increased levels of inflammatory mediators such as proinflammatory cytokines (IL-6 and IL-1 $1 \beta$ ) have been reported in diabetic states to be a consequence of hyperglycemia (Brownlee, 2005) and these mediators have been considered to be the link between inflammation and insulin resistance (Navarro-Gonzalez and Mora-Fernandez, 2008). In present study, serum proinflammatory markers including TNF- $\alpha$, IL- 6 and IL-1 $\beta$ are significantly increased in STZ-induced diabetic rats. Rutin treatment in diabetic rats significantly reduced such markers in the present study. This may be because rutin showed antioxidant and anti-inflammatory properties in 

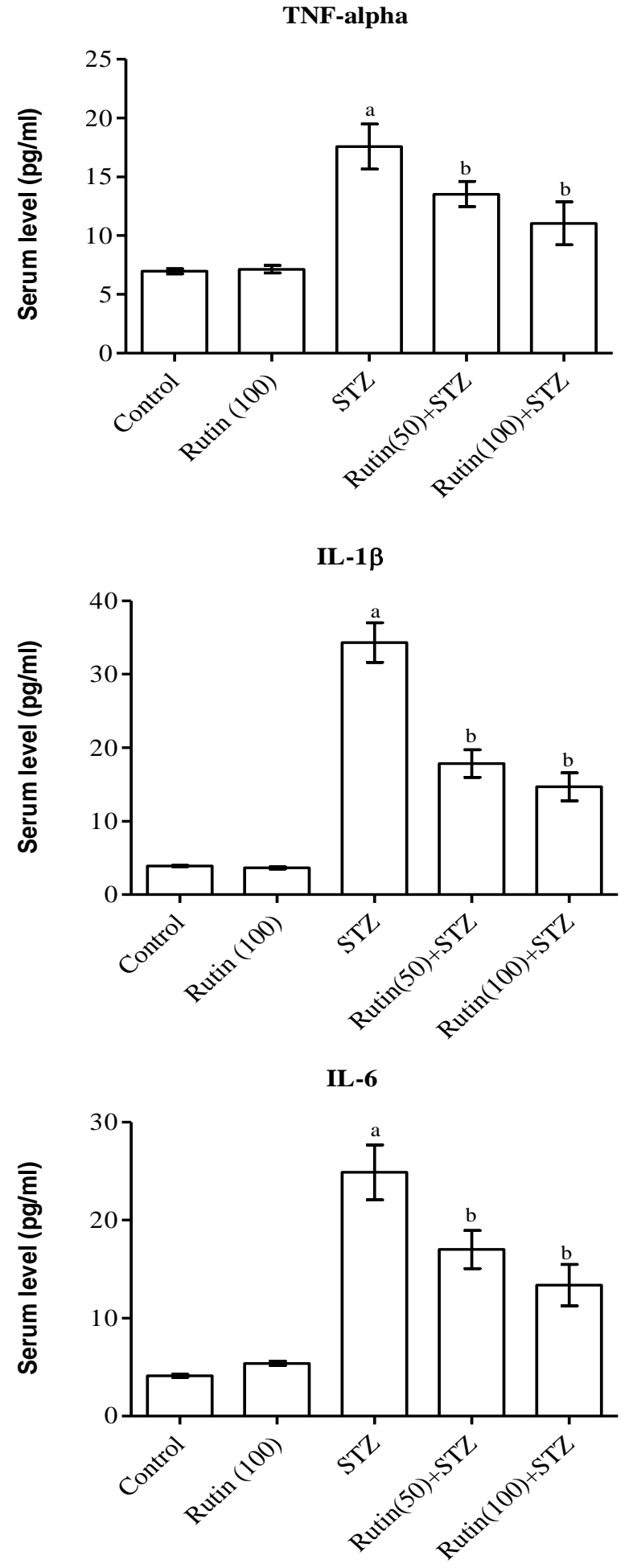

Figure 4. Effect of rutin on serum TNF- $\alpha, I L-1 \beta$ and IL- 6 levels of diabetic and non-diabetic rats. One-way ANOVA and Student-Newman-Keuls multiple comparisons test was applied.

aSignificantly different from control group $(P<0.05)$ and ${ }^{b}$ Significantly different from STZ group $(P<0.05)$. Values are expressed as Mean \pm SEM and six rats were used in each group. earlier studies (Abu-El-Fattah et al., 2010; Alsaif, 2009). Kwon et al. (2005) reported that, rutin ameliorates dextran sulfate sodium-induced experimental colitis in mice by following attenuation of pro-inflammatory gene expressions.

Chronic hyperglycemia induces oxidative stress by the autoxidation of monosaccharides (Bonnefont-Rousselot, 2002), which leads to production of superoxide and hydroxyl radicals. It is well known that pain transmission requires production of reactive oxygen species (Viggiano et al., 2005). We observed a significantly higher level of lipid peroxidation marker, MDA, in sciatic nerve of diabetic animals. Glutathione, a potent endogenous antioxidant is a first line of defense against free radicals. The GSH levels were significantly lowered in the sciatic nerve of diabetic animals. These observations are in agreement with previous findings showing reduction in GSH levels in diabetes (Kuzumoto et al., 2006; Arora et al., 2008). Intracellular GSH levels have been observed to decrease in brain (Kamboj et al., 2008) and sciatic nerve (Kuzumoto et al., 2006) of diabetic animals. Rutin treatment significantly reduced lipid peroxidation and regenerated intracellular GSH content in the sciatic nerve; this is probably because of its free radical scavenging activity or endogenous synthesis of GSH induced by rutin.

The results of the present study are in agreement with earlier studies wherein decreased SOD activity was observed in nerves isolated from diabetic rats (Cui et al., 2008). SOD and CAT are major antioxidant enzymes involved in protection from oxidative stress. SOD offers protection from highly reactive superoxide anions $\left(\mathrm{O}_{2}{ }^{-}\right)$ and converts them to $\mathrm{H}_{2} \mathrm{O}_{2}$ (Halliwell, 1991). Hyperglycemia caused reduction in the activity of SOD in sciatic nerve of diabetic animals. Reduction in SOD activity in hyperglycemia might involve non-enzymatic glycosylation (Arai et al., 1987). Increased SOD activity after rutin administration to the diabetic animals is in accordance with reported restoration of SOD activity by rutin in hepatic cells (Kamalakkannan and Prince, 2006) and kidney (Alsaif, 2009). CAT is responsible for the catalytic decomposition of $\mathrm{H}_{2} \mathrm{O}_{2}$ to $\mathrm{O}_{2}$ and $\mathrm{H}_{2} \mathrm{O}$. The decreased CAT activity in diabetes might reduce protection against free radicals. It is clear that the simultaneous reduction in the activity of both SOD and CAT makes the sciatic nerve more vulnerable to hyperglycemia-induced oxidative stress. Reports are available wherein rutin has been shown to bring about improvement in the CAT activity during diabetic-induced nephrotoxicity in rats (Alsaif, 2009). The results obtained emphasize that rutin protects the sciatic nerve from hyperglycemia induced damage by restoring the activity of both these enzymes. Glutathione reductase is an important enzyme involved in maintaining high GSH/GSSG ratios (Carlberg and Mannervik, 1985). Present data showed a significant decrease in the activity of GR in sciatic nerve of diabetic 


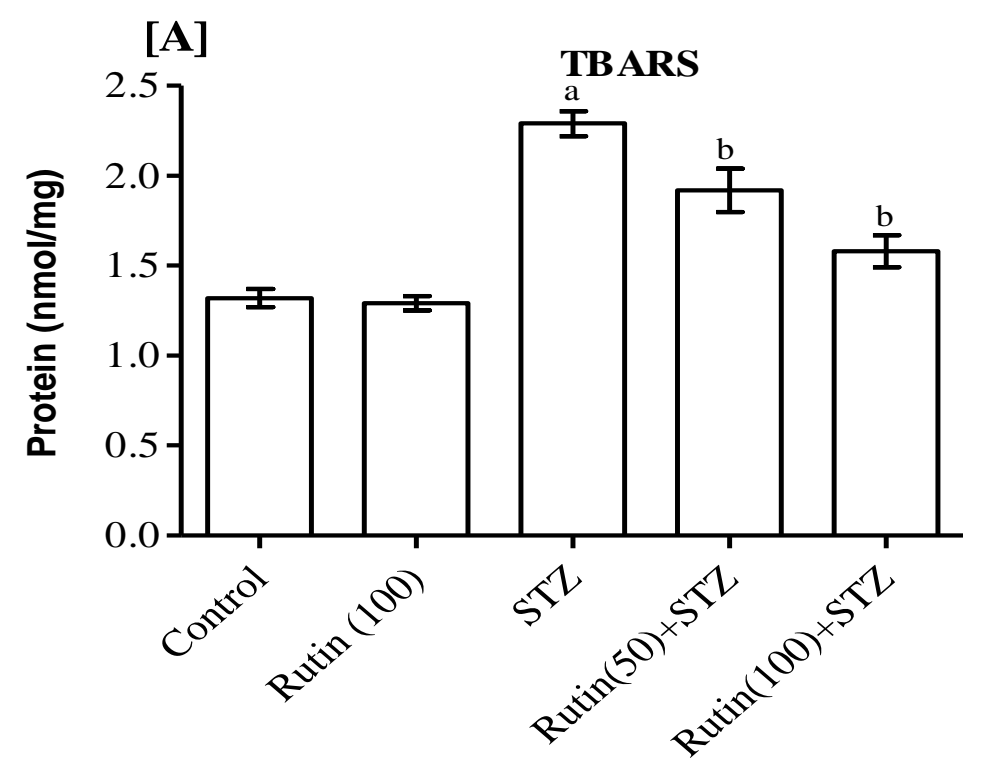

[B]

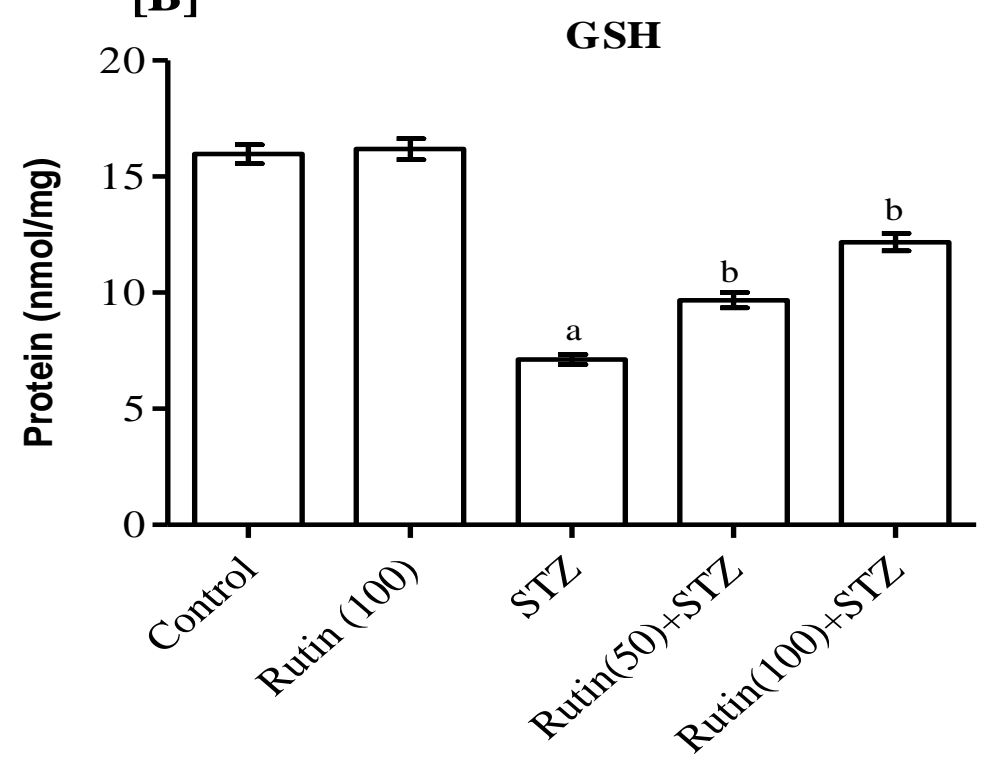

Figure 5. Effect of rutin on TBARs and GSH levels in sciatic nerve of diabetic and non-diabetic rats. One-way ANOVA and Student-NewmanKeuls multiple comparisons test was applied. ${ }^{a}$ Significantly different from control group $(\mathrm{P}<0.05)$ and ${ }^{b}$ Significantly different from STZ group $(\mathrm{P}<0.05)$. Values are expressed as Mean $\pm \mathrm{SEM}$ and six rats were used in each group.

animals. The results obtained from the earlier studies also showed depressed GR activity in sciatic nerve (Kamboj et al., 2010) and increase and decrease in brain and other organs of diabetic animals (Ulusu et al., 2003; Sanders et al., 2001). The reversal of GR activity by rutin treatment might result in increasing intracellular $\mathrm{GSH}$ levels. Previous studies have shown that rutin protects against the reproductive toxicity effects of cyclophosphamide by increasing GR activity (Abarikwu et al., 2012). In another study, Kamalakkannan and Prince (2006) reported that, rutin treatment to diabetic rats significantly enhanced theGR activity in hepatic cells. In the present studies, the activity of GSH-Px was found to be significantly depressed in sciatic nerve of diabetic rats. This decrease in GSH-Px activity was reversed by the rutin treatment. 

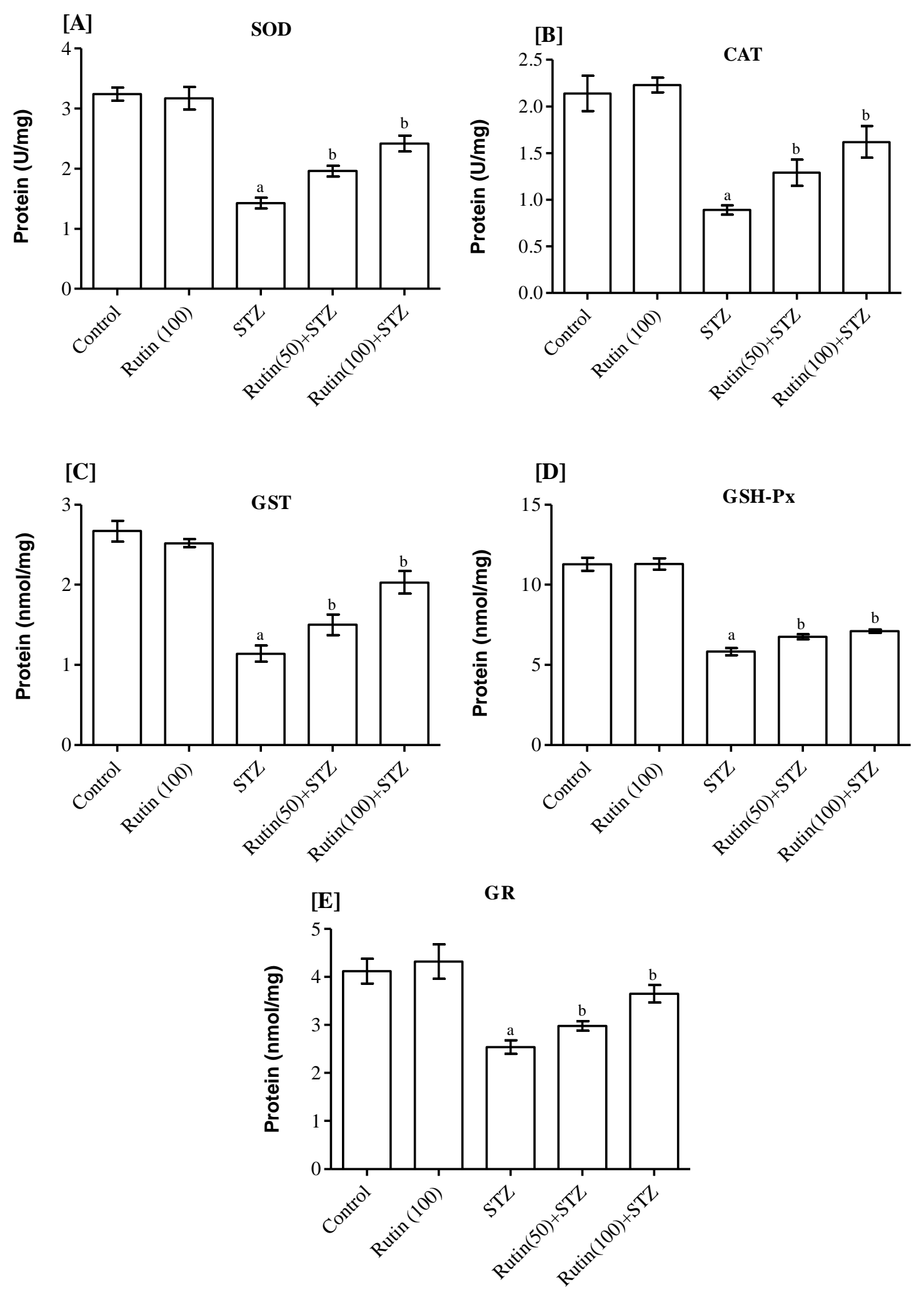

Figure 6. Effect of rutin on SOD, CAT, GST, GST-Px and GR activities in sciatic nerve of diabetic and nondiabetic rats. One-way ANOVA and Student-Newman-Keuls multiple comparisons test was applied. 'a' Significantly different from control group $(\mathrm{P}<0.05)$ and 'b' Significantly different from STZ group $(\mathrm{P}<0.05)$. Values are expressed as Mean \pm SEM and six rats were used in each group. 

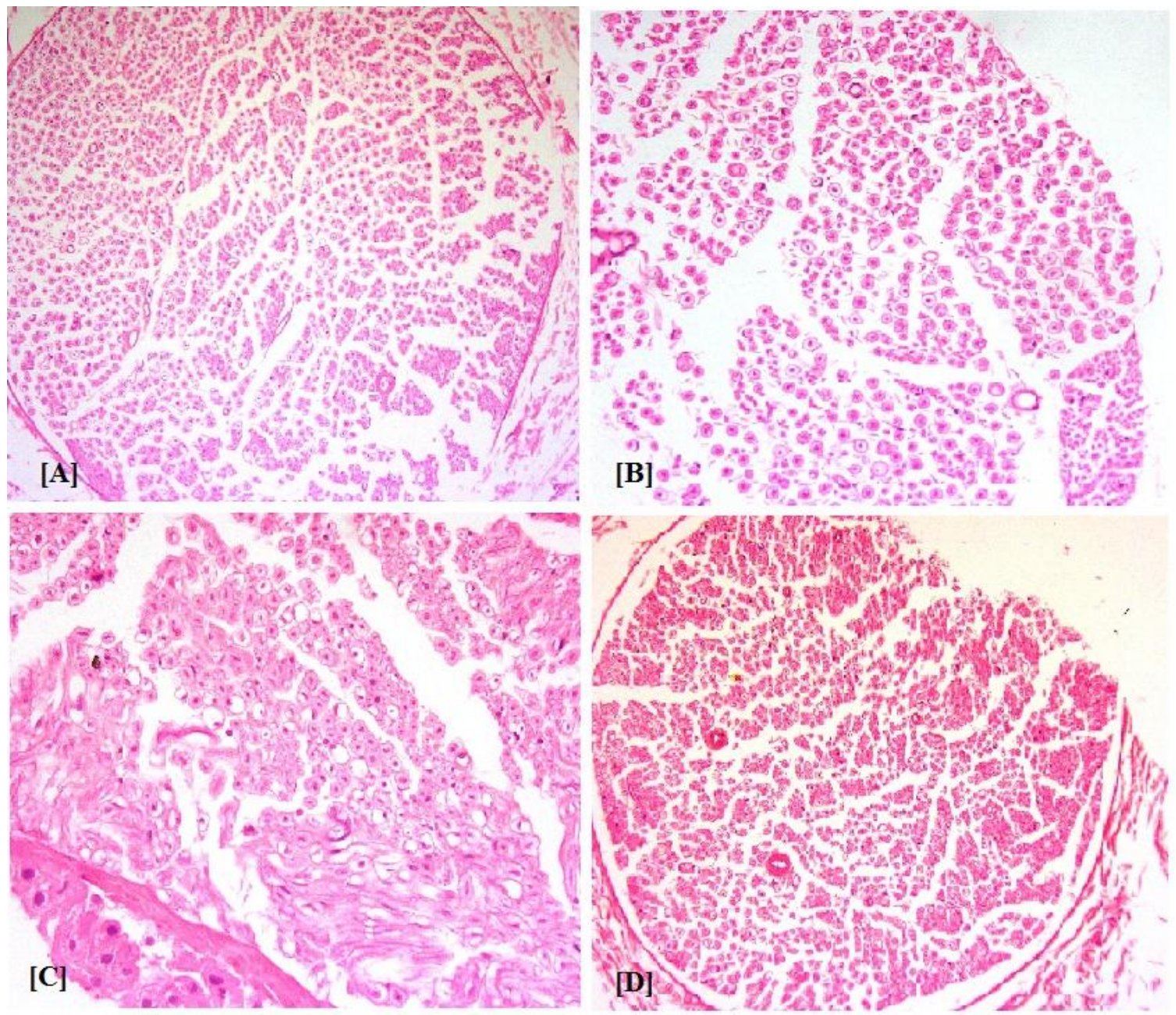

Figure 7. $[A]$ Rat transverse section of sciatic nerve from control group looks normal, $[B]$ in diabetic rat, sever nerve damage was seen, $[\mathrm{C}]$ rutin $(50 \mathrm{mg} / \mathrm{kg} /$ day $)$ treatment to diabetic rats produced mild protection in sciatic nerve, and [D] in higher dose $(100 \mathrm{mg} / \mathrm{kg} / \mathrm{day})$ of rutin treatment to diabetic rats showing normal look similar to control.

Glutathione reductase is responsible for the regeneration of GSH, whereas GSH-Px and GST work together with GSH in the decomposition of $\mathrm{H}_{2} \mathrm{O}_{2}$ or other organic hydroperoxides. A reduction observed in sciatic nerve GR, GSH-Px and GST activity in diabetic rats might be reflection of decreased protein thiols observed in the study as -SH groups play a critical role in enzyme catalysis (Mak et al., 1996). Rutin treatment ameliorates decrease in the activity of these enzymes which might be mediated by GSH regeneration.

In conclusion, results obtained from the present study revealed that rutin ameliorates hyperglycemia-induced thermal hyperalgesia and improves the neuropathic pain by reducing oxidative stress in the nerve of diabetic rats by virtue of its antioxidative properties. Morphological assessments also show that the damage caused by streptozotocin to the sciatic nerve was also markedly reduced by the administration of rutin. Finally, these findings suggest that rutin treatment might be beneficial in chronic diabetics exhibiting neuropathy.

\section{ACKNOWLEDGEMENTS}

This study was funded by the Deanship of Scientific Research at Al-Jouf University through the research group project No. 1-32. The investigator kind heartedly appreciates and acknowledged the Experimental Animal Care Center, College of Pharmacy, King Saud University, Riyadh for supplying the experimental animals.

\section{REFERENCES}

Abarikwu SO, Otuechere CA, Ekor M, Monwuba K, Osobu D (2012). Rutin Ameliorates Cyclophosphamide-induced Reproductive Toxicity in Male Rats. Toxicol. Int. 19:207-214. 
Abdi S, Haruo A, Bloomstone J (2004). Electroconvulsive therapy for neuropathic pain: a case report and literature review. Pain Physician 7:261-263.

Abu-El-Fattah AA, El-Sawalhi MM, Rashed ER, El-Ghazaly MA (2010). Possible role of vitamin E, coenzyme Q10 and rutin in protection against cerebral ischemia/reperfusion injury in irradiated rats. Int. J. Radiat. Biol. 86:1070-1078.

Aebi H (1094). Catalase In: Bergmeyer, editor. Methods in enzymatic analysis. New York, pp. 674-684.

Al-Enazi MM (2013). Neuroprotective effect of silymarin by modulation of endogenous biomarkers in streptozotocin induced painful diabetic neuropathy. Br. J. Pharmacol. Toxicol. 4:110-120.

Alsaif MA (2009). Beneficial Effects of Rutin and Vitamin C Coadministration in a Streptozotocin-Induced Diabetes Rat Model of Kidney Nephrotoxicity. Pak. J. Nutr. 8:745-754.

Apfel SC, Asbury AK, Bril V, Burns TM, Campbell JN, Chalk CH, Dyck PJ, Dyck PJ, Feldman EL, Fields HL, Grant IA, Griffin JW, Klein CJ, Lindblom U, Litchy WJ, Low PA, Melanson M, Mendell JR, Merren MD, O'Brien PC, Rendell M, Rizza RA, Service FJ, Thomas PK, Walk D, Wang AK, Wessel K, Windebank AJ, Ziegler D, Zochodne DW (2001). Positive neuropathic sensory symptoms as endpoints in diabetic neuropathy trials. J. Neurol. Sci. 189:3-5.

Arai K, Maguchi S, Fujii S, Ishibashi H, Oikawa K, Taniguchi N (1987). Glycation and inactivation of human $\mathrm{Cu}-\mathrm{Zn}$-superoxide dismutase. Identification of the in vitro glycated sites. J. Biol. Chem. 262:1696916972.

Arora M, Kumar A, Kaundal RK, Sharma SS (2008). Amelioration of neurological and biochemical deficits by peroxynitrite decomposition catalysts in experimental diabetic neuropathy. Eur. J. Pharmacol. 596:77-83

Bierhaus A, Ziegler R, Nawroth PP (1998). Molecular mechanisms of diabetic angiopathy--clues for innovative therapeutic interventions. Horm. Res. 50 (Suppl 1):1-5.

Bonnefont-Rousselot D (2002). Glucose and reactive oxygen species. Curr. Opin. Clin. Nutr. Metab. Care 5:561-568.

Brownlee M (2005). The pathobiology of diabetic complications: a unifying mechanism. Diabetes 54:1615-1625.

Calcutt NA (2004). Experimental models of painful diabetic neuropathy. J. Neurol. Sci. 220:137-139.

Callaghan BC, Little AA, Feldman EL, Hughes RA (2012). Enhanced glucose control for preventing and treating diabetic neuropathy. Cochrane Database Syst. Rev. 6: CD007543.

Cameron NE, Cotter MA (1999). Effects of antioxidants on nerve and vascular dysfunction in experimental diabetes. Diabetes Res. Clin. Pract. 45:137-146.

Carlberg I, Mannervik B (1985). Glutathione reductase. Methods Enzymol. 113:484-490.

Cartmell SM, Gelgor L, Mitchell D (1991). A revised rotarod procedure for measuring the effect of antinociceptive drugs on motor function in the rat. J. Pharmacol. Methods 26:149-159.

Chen X, Levine JD (2001). Hyper-responsivity in a subset of C-fiber nociceptors in a model of painful diabetic neuropathy in the rat. Neuroscience 102:185-192.

Choi I, Park Y, Choi H, Lee EH (2006). Anti-adipogenic activity of rutin in 3T3-L1 cells and mice fed with high-fat diet. Biofactors 26:273-281.

Cui XP, Li BY, Gao HQ, Wei N, Wang WL, Lu M (2008). Effects of grape seed proanthocyanidin extracts on peripheral nerves in streptozocin-induced diabetic rats. J. Nutr. Sci. Vitaminol. (Tokyo) 54:321-328

Cunha JM, Jolivalt CG, Ramos KM, Gregory JA, Calcutt NA, Mizisin AP (2008). Elevated lipid peroxidation and DNA oxidation in nerve from diabetic rats: effects of aldose reductase inhibition, insulin, and neurotrophic factors. Metabolism 57:873-881.

Dobretsov M, Hatings SL, Romanovsky D, Stimers JR, Zhang JM (2003). Mechanical hyperalgesia in rat models of systemic and local hyperglycemia. Brain Res. 960:174-183.

Edwards JL, Vincent AM, Cheng HT, Feldman EL (2008). Diabetic neuropathy: mechanisms to management. Pharmacol. Ther. 120:134.

Figueroa-Romero C, Sadidi M, Feldman EL (2008). Mechanisms of disease: the oxidative stress theory of diabetic neuropathy. Rev. Endocr. Metab. Disord. 9:301-314.

Flohe L, Gunzler WA (1984). Assays of glutathione peroxidase. Methods Enzymol. 105:114-121.

Fuchs D, Birklein F, Reeh PW, Sauer SK (2010). Sensitized peripheral nociception in experimental diabetes of the rat. PAIN 151:496-505.

Galer BS, Gianas A, Jensen MP (2000). Painful diabetic polyneuropathy: epidemiology, pain description, and quality of life. Diabetes Res. Clin. Pract. 47:123-128.

Gilron I, Watson CP, Cahill CM, Moulin DE (2006). Neuropathic pain: a practical guide for the clinician. CMAJ 175:265-275

Goldberg RB (2009). The Endocrine Society, Cytokine and CytokineLike Inflammation Markers, Endothelial Dysfunction, and Imbalanced Coagulation in Development of Diabetes and Its Complications. J. Clin. Endocrinol. Met. 94:3171-3182.

Gul H, Yildiz O, Dogrul A, Yesilyurt O, Isimer A (2000). The interaction between IL-1beta and morphine: possible mechanism of the deficiency of morphine-induced analgesia in diabetic mice. Pain 89:39-45.

Habig WH, Pabst MJ, Jakoby WB (1974). Glutathione S-transferases. The first enzymatic step in mercapturic acid formation. J. Biol. Chem. 249:7130-7139.

Halliwell B (1991). Drug antioxidant effects. A basis for drug selection? Drugs 42:569-605.

Hao HH, Shao ZM, Tang DQ, Lu Q, Chen X, Yin XX, Wu J, Chen $\mathrm{H}$ (2012). Preventive effects of rutin on the development of experimental diabetic nephropathy in rats. Life Sci. 91(19-20):959967.

Inme N, Kiesewetter $\quad H$, Jung $F$, Hoffmann $\quad K H$, Birk A, Müller A, Grützner KI (1996). Leg oedema protection from a buckwheat herb tea in patients with chronic venous insufficiency: a single-centre, randomised, double-blind, placebo-controlled clinical trial. Eur. J. Clin. Pharmacol. 50:443-447.

Kamalakkannan N, Prince PS (2006). Antihyperglycaemic and antioxidant effect of rutin, a polyphenolic flavonoid, in streptozotocininduced diabetic wistar rats. Basic Clin. Pharmacol. Toxicol. 98:97103.

Kamboj SS, Vasishta RK, Sandhir R (2010). N-acetylcysteine inhibits hyperglycemia-induced oxidative stress and apoptosis markers in diabetic neuropathy. J. Neurochem. 112:77-91.

Kamei J, Zushida K, Morita K, Sasaki M, Tanaka S (2001). Role of vanilloid VR1 receptor in thermal allodynia and hyperalgesia in diabetic mice. Eur. J. Pharmacol. 422:83-86.

Kono Y (1978). Generation of superoxide radical during autoxidation of hydroxylamine and an assay for superoxide dismutase. Arch. Biochem. Biophys. 186:189-195.

Kuzumoto Y, Kusunoki S, Kato N, Kihara M, Low PA (2006). Effect of the aldose reductase inhibitor fidarestat on experimental diabetic neuropathy in the rat. Diabetologia 49:3085-3093.

Kwon KH, Murakami A, Tanaka T, Ohigashi H (2005). Dietary rutin, but not its aglycone quercetin, ameliorates dextran sulfate sodiuminduced experimental colitis in mice: attenuation of pro-inflammatory gene expression. Biochem. Pharmacol. 69:395-406.

Lee JY, Jang YW, Kang HS, Moon H, Sim SS, Kim CJ (2006). Antiinflammatory action of phenolic compounds from Gastrodia elata root. Arch. Pharm. Res. 29:849-858.

Lindahl M, Tagesson C (1997). Flavonoids as phospholipase A2 inhibitors: importance of their structure for selective inhibition of group II phospholipase A2. Inflammation 21:347-356.

Locksley RM, Killeen N, Lenardo MJ (2001). The TNF and TNF receptor superfamilies: integrating mammalian biology. Cell 104:487-501.

Lopez-Revuelta A, Sanchez-Gallego JI, Hernandez-Hernandez A, Sanchez-Yague J, Llanillo M (2006). Membrane cholesterol contents influence the protective effects of quercetin and rutin in erythrocytes damaged by oxidative stress. Chem. Biol. Interact. 161:79-91.

Mak DH, Ip SP, Li PC, Poon MK, Ko KM (1996). Alterations in tissue glutathione antioxidant system in streptozotocin-induced diabetic rats. Mol. Cell Biochem. 162:153-158.

Mora C, Navarro JF (2006). Inflammation and diabetic nephropathy. Curr. Diabetes Rep. 6:463-468. 
Navarro-Gonzalez JF, Mora-Fernandez C (2008). The role of inflammatory cytokines in diabetic nephropathy. J. Am. Soc. Nephrol. 19:433-442.

Park SY, Bok SH, Jeon SM, Park YB, Lee SJ, Jeong TS, Choi MS (2002). Effect of rutin and tannic acid supplements on cholesterol metabolism in rats. Nutr. Res. 22:283-295.

Ramirez MR, Guterres L, Dickel OE, de Castro MR, Henriques AT, de Souza MM, Barros DM (2010). Preliminary studies on the antinociceptive activity of Vaccinium ashei berry in experimental animal models. J. Med. Food. 13:336-342.

Romanovsky D, Hastings SL, Stimers JR, Dobretsov M (2004). Relevance of hyperglycemia to early mechanical hyperalgesia in streptozotocin-induced diabetes. J. Peripher. Nerv. 9:62-69.

Sedlak J, Lindsay RH (1968). Estimation of total, protein-bound, and nonprotein sulfhydryl groups in tissue with Ellman's reagent. Anal. Biochem. 25:192-205.

Shen SC, Lee WR, Lin HY, Huang HC, Ko CH, Yang LL, Chen YC, (2002). In vitro and in vivo inhibitory activities of rutin, wogonin, and quercetin on lipopolysaccharide-induced nitric oxide and prostaglandin $E(2)$ production. Eur. J. Pharmacol. 446:187-194.

Sima AA, Zhang W, Xu G, Sugimoto K, Guberski D, Yorek MA (2000). A comparison of diabetic polyneuropathy in type II diabetic BBZDR/Wor rats and in type I diabetic BB/Wor rats. Diabetologia 43: 786-793.

Stevens MJ, Obrosova I, Cao X, Van Huysen C, Greene DA (2000). Effects of DL-alpha-lipoic acid on peripheral nerve conduction, blood flow, energy metabolism, and oxidative stress in experimental diabetic neuropathy. Diabetes 49:1006-1015.

Sugimoto K, Rashid IB, Shoji M, Suda T, Yasujima M (2008). Early changes in insulin receptor signaling and pain sensation in streptozotocin-induced diabetic neuropathy in rats. J. Pain 9:237-245.

Tuttle KR (2005). Linking metabolism and immunology Diabetic nephropathy is an inflammatory disease. J. Am. Soc. Nephrol. 16:1537-1538.
Ulusu NN, Sahilli M, Avci A, Canbolat O, Ozansoy G, Ari N, Bali M, Stefek M, Stolc S, Gajdosik A, Karasu C (2003). Pentose phosphate pathway, glutathione-dependent enzymes and antioxidant defense during oxidative stress in diabetic rodent brain and peripheral organs: effects of stobadine and vitamin E. Neurochem. Res. 28:815-823.

Viggiano A, Monda M, Viggiano A, Viggiano D, Viggiano E, Chiefari M, Aurilio C, De Luca B (2005). Trigeminal pain transmission requires reactive oxygen species production. Brain Res. 1050:72-78.

Wang S, Li Y, Zhao J, Zhang J, Huang Y (2013). Mesenchymal stem cells ameliorate podocyte injury and proteinuria in a type 1 diabetic nephropathy rat model. Biol. Blood Marrow Transplant. 19:538-546.

Woolf CJ (2004). Dissecting out mechanisms responsible for peripheral neuropathic pain: implications for diagnosis and therapy. Life Sci. 74:2605-2610.

Yu J, Zhang Y, Sun S, Shen J, Qiu J, Yin X, Yin H, Jiang S (2006) Inhibitory effects of astragaloside IV on diabetic peripheral neuropathy in rats. Can. J. Physiol. Pharmacol. 84:579-587.

Zherebitskaya E, Akude E, Smith DR, Fernyhough P (2009). Development of selective axonopathy in adult sensory neurons isolated from diabetic rats: role of glucose-induced oxidative stress. Diabetes 58:1356-1364

Sanders RA, Rauscher FM, Watkins JB (2001). Effects of quercetin on antioxidant defense in streptozotocin-induced diabetic rats. J. Biochem. Mol. Toxicol. 15:143-149. 